\title{
Simplified dark matter models in the light of AMS-02 antiproton data
}

\author{
Tong Li \\ ARC Centre of Excellence for Particle Physics at the Tera-scale, \\ School of Physics and Astronomy, Monash University, \\ Melbourne, Victoria 3800 Australia \\ E-mail: tong.li@monash.edu
}

ABSTRACT: In this work we perform an analysis of the recent AMS-02 antiproton flux and the antiproton-to-proton ratio in the framework of simplified dark matter models. To predict the AMS-02 observables we adopt the propagation and injection parameters determined by the observed fluxes of nuclei. We assume that the dark matter particle is a Dirac fermionic dark matter, with leptophobic pseudoscalar or axialvector mediator that couples only to Standard Model quarks and dark matter particles. We find that the AMS02 observations are consistent with the dark matter framework within the uncertainties. The antiproton data prefer a dark matter (mediator) mass in the $700 \mathrm{GeV}-5 \mathrm{TeV}$ region for the annihilation with pseudoscalar mediator and greater than $700 \mathrm{GeV}(200 \mathrm{GeV}-1 \mathrm{TeV})$ for the annihilation with axialvector mediator, respectively, at about $68 \%$ confidence level. The AMS-02 data require an effective dark matter annihilation cross section in the region of $1 \times 10^{-25}-1 \times 10^{-24}\left(1 \times 10^{-25}-4 \times 10^{-24}\right) \mathrm{cm}^{3} / \mathrm{s}$ for the simplified model with pseudoscalar (axialvector) mediator. The constraints from the LHC and Fermi-LAT are also discussed.

KEYwords: Beyond Standard Model, Cosmology of Theories beyond the SM

ArXiv EPrint: 1612.09501 


\section{Contents}

1 Introduction 1

2 Propagation and injection of cosmic rays 2

3 The simplified dark matter models 4

4 Results $\quad 6$

5 Conclusions 12

A Expressions of mediator decay widths and dark matter annihilation cross sections

\section{Introduction}

The charged cosmic rays provide abundant information connecting the galactic astrophysics with possibly new fundamental particle physics. Deciphering the information is, however, challenging to our understanding of nature, from basic matter structure, fundamental interactions to cosmology. The cosmic rays arriving at our earth not only include the products during the long journey from the astrophysical sources, but also a certain fraction may be attributed to more exotic new physics such as dark matter particles which convert into the standard matter. Therefore a careful analysis of the constitution and propagation of cosmic rays may let us get knowledge about the dark matter.

The recent data on the cosmic ray nuclei observed by the AMS-02 collaboration, e.g. proton [1], antiproton [2], Helium [3], etc., can help to determine the propagation/source injection parameters with high precision and moreover, confine the leptophobic dark matter models. These measurements have drawn the great attention of both astrophysicists and particle physicists [4-11].

The propagation parameters can be determined by fitting the secondary-to-primary ratio of cosmic ray nuclei, such as the Boron-to-Carbon ratio $(\mathrm{B} / \mathrm{C})$, and the ratio of secondary nuclei, such as the Beryllium isotope ratio ${ }^{10} \mathrm{Be} /{ }^{9} \mathrm{Be}$. The observed proton flux can further fix the unified injection parameters of all nuclei. Based on these obtained parameters, one can derive an up-to-date astrophysical background for the secondary production of antiprotons. Then we are enabled to study extra compositions in cosmic rays which are associated with dark matter. A self-consistent approach to take the dark matter into account as an extra source is to propagate the antiproton spectrum induced by dark matter annihilation through the Galaxy and calculate the antiproton flux in terms of the same astrophysical parameters. This procedure ensures consistency between astrophysical treatments of cosmic rays coming from standard astrophysical sources and that concerning dark matter [12]. 
In this work, we examine the constraint set by the AMS-02 data of antiproton flux and antiproton-to-proton ratio on the simplified models with leptophobic dark matter. This framework is widely used to analyze the data for dark matter search at the Large Hadron Collider (LHC), the satellites in the sky and the underground direct detection experiments [13-19]. It uses minimal and general theoretical assumptions with only two parameters, i.e. the dark matter mass and the mediator mass. The simultaneous presence of various annihilation channels provides the dark matter models with considerable flexibility. We specifically consider a Dirac fermionic dark matter, with pseudoscalar and axialvector mediators that only couple to the Standard Model (SM) quarks and dark matter particles. The dark matter annihilations are not velocity suppressed [20]. Meanwhile, the dark matter-nucleon elastic scattering cross sections are spin-dependent (SD) thus the models do not receive stringent constraint from direct detection. For these dark matter models, we derive the AMS-02 preferred region in the parameter space.

This paper is organized as follows. In section 2 we describe the propagation equation and injection spectra for cosmic ray nuclei. The values of corresponding parameters are also given. In section 3, we describe the simplified dark matter models we use. Our numerical results are given in section 4 . Finally, in section 5 we summarize our conclusions.

\section{Propagation and injection of cosmic rays}

Cosmic rays in the Galaxy are categorized into primary and secondary types [21-24]. Primary cosmic rays are stable charged particles that are accelerated to high energies by astrophysical sources in the universe. After being accelerated near the sources, Galactic cosmic ray particles propagate in the interstellar medium (ISM) and undergo the processes of decay, fragmentation, energy loss and possible reacceleration or convection. Their initial spectra and composition thus change and they produce secondary cosmic ray particles. The propagation of cosmic rays can thus be described as the process of diffusion. The diffusion process also explains the retention and isotropic distribution of high energy charged particles within the Galaxy.

The cosmic ray propagation within the galaxy is described by the following transport equation [25]

$$
\begin{aligned}
\frac{\partial \psi}{\partial t}= & Q(\vec{r}, p)+\vec{\nabla} \cdot\left(D_{x x} \vec{\nabla} \psi-\vec{V} \psi\right)+\frac{\partial}{\partial p} p^{2} D_{p p} \frac{\partial}{\partial p} \frac{1}{p^{2}} \psi \\
& -\frac{\partial}{\partial p}\left[\dot{p} \psi-\frac{p}{3}(\vec{\nabla} \cdot \vec{V}) \psi\right]-\frac{\psi}{\tau_{f}}-\frac{\psi}{\tau_{r}}
\end{aligned}
$$

where $\psi(\vec{r}, t, p)$ is the density of cosmic rays per unit of total particle momentum $p . \vec{V}$ is the convection velocity and $\tau_{f}\left(\tau_{r}\right)$ is the time scale for fragmentation (radioactive decay). $\dot{p}$ is the momentum loss rate. The convection terms in the above equation are induced by the Galactic wind. The diffusion in momentum space governs the reacceleration process. In this case the diffusion coefficient in momentum space, i.e. $D_{p p}$, is related to the spatial coefficient $D_{x x}$ and the Alfven velocity $v_{A}[26]$ :

$$
D_{p p} D_{x x}=\frac{4 p^{2} v_{A}^{2}}{3 \delta\left(4-\delta^{2}\right)(4-\delta) w}
$$


with the level of the interstellar turbulence parameter $w$ being 1 . The spatial diffusion coefficient is usually written in this form

$$
D_{x x}=\beta D_{0}\left(R / R_{0}\right)^{\delta},
$$

with $R$ and $\beta$ being the rigidity and particle velocity divided by light speed respectively. This transport equation is numerically solved based on given boundary conditions, that is, the cosmic ray density $\psi$ vanishes at the radius $R_{h}$ and the height $z_{0}$ of the cylindrical diffusion halo.

The above key propagation parameters can be constrained by fitting the latest ratios of nuclei, that is the Boron-to-Carbon ratio $(\mathrm{B} / \mathrm{C})$ and the Beryllium ratio $\left({ }^{10} \mathrm{Be} /{ }^{9} \mathrm{Be}\right)$. We adopt the values of propagation parameters shown in table 1 , determined by the $\mathrm{B} / \mathrm{C}$ and ${ }^{10} \mathrm{Be} /{ }^{9} \mathrm{Be}$ data [6]. The benchmark model of the propagation we use is diffusion reacceleration model. The ${ }^{10} \mathrm{Be} /{ }^{9} \mathrm{Be}$ ratio data are sensitive to the diffusion halo size $z_{0}$. For a given halo size the diffusion coefficient as a function of momentum and the reacceleration parameter are determined by $\mathrm{B} / \mathrm{C}$ ratio data. The values in table 1 are the best fit results. The diffusion reacceleration scenario is found to be best consistent with the data compared with other propagation scenarios, such as the diffusion convection model [27].

In eq. (2.1), the source term of cosmic ray species $i$ can be described by the product of the spatial distribution and the injection spectrum function

$$
Q_{i}(\vec{r}, p)=f(r, z) q_{i}(p)
$$

For the spatial distribution of the primary cosmic rays we use the following supernova remnants distribution

$$
f(r, z)=f_{0}\left(\frac{r}{r_{\odot}}\right)^{a} \exp \left(-b \frac{r-r_{\odot}}{r_{\odot}}\right) \exp \left(-\frac{|z|}{z_{s}}\right),
$$

where $r_{\odot}=8.5 \mathrm{kpc}$ is the distance between the Sun and the Galactic center, the height of the Galactic disk is $z_{s}=0.2 \mathrm{kpc}$, and the two parameters $a$ and $b$ are chosen to be 1.25 and 3.56 , respectively [27]. We assume the following power law with one break for the injection spectrum of various nuclei

$$
q_{i} \propto\left\{\begin{array}{l}
\left(R / R_{\mathrm{br}}^{p}\right)^{-\nu_{1}}, R \leq R_{\mathrm{br}}^{p} \\
\left(R / R_{\mathrm{br}}^{p}\right)^{-\nu_{2}}, R>R_{\mathrm{br}}^{p}
\end{array}\right.
$$

The corresponding injection parameters in eq. (2.6), i.e. rigidity break $R_{\mathrm{br}}^{p}$ and power law indexes $\nu_{1}, \nu_{2}$, can be determined by fitting the latest AMS-02 proton data [1]. We adopt injection parameters obtained by performing such a fit in ref. [6]. The values of these injection parameters are shown in table 1 , together with the Fisk potential $\phi_{i}(i=p, \bar{p})$ for solar modulation effect. The values of Fisk potential come from ref. [6]. They are the approximate constants of the time-varying modulation form employed in the reference. 


\begin{tabular}{|c|c|c|c|c|c|}
\hline Propagation & Value & Nucleon injection & Value & Solar modulation & Value \\
\hline$D_{0}\left(10^{28} \mathrm{~cm}^{2} \mathrm{~s}^{-1}\right)$ & 7.09 & $\nu_{1}$ & 1.702 & $\phi_{p}(\mathrm{MV})$ & 550 \\
\hline$\delta$ & 0.349 & $\nu_{2}$ & 2.399 & $\phi_{\bar{p}}(\mathrm{MV})$ & 400 \\
\hline$R_{0}(\mathrm{GV})$ & 4 & $R_{\mathrm{br}}^{p}(\mathrm{GV})$ & 11.48 & - & - \\
\hline$v_{A}\left(\mathrm{~km} \mathrm{~s}^{-1}\right)$ & 38.14 & $A_{p}($ see caption $)$ & 4.325 & - & - \\
\hline$z_{0}(\mathrm{kpc})$ & 5.47 & - & - & - & - \\
\hline
\end{tabular}

Table 1. Parameters of propagation, nucleon injection and solar modulation and their values adopted in our numerical analysis. The proton flux is normalized to $A_{p}$ at $100 \mathrm{GeV}$ in the units of $10^{-9} \mathrm{~cm}^{-2} \mathrm{~s}^{-1} \mathrm{sr}^{-1} \mathrm{MeV}^{-1}$.

\section{The simplified dark matter models}

In this section, we describe the simplified dark matter models restricted by the AMS-02 data of antiproton flux and antiproton-to-proton ratio. We assume that dark matter is composed of Dirac fermionic particles, which we denote by $\chi$. The dark matter particles couple to the SM quarks through a pseudoscalar mediator $S$ or an axialvector mediator $V$. The corresponding interactions are as follows [16]

$$
\begin{aligned}
\mathcal{L}_{\text {pseudoscalar }} & =-i g_{\mathrm{DM}}^{S} S \bar{\chi} \gamma_{5} \chi-i g_{q}^{S} S \sum_{q=u, d, s, c, b, t} \frac{m_{q}}{v_{0}} \bar{q} \gamma_{5} q, \\
\mathcal{L}_{\text {axialvector }} & =-g_{\mathrm{DM}}^{A} V_{\mu} \bar{\chi} \gamma^{\mu} \gamma_{5} \chi-g_{q}^{A} V_{\mu} \sum_{q=u, d, s, c, b, t} \bar{q} \gamma^{\mu} \gamma_{5} q,
\end{aligned}
$$

where $v_{0}=246 \mathrm{GeV}$ is the Higgs vacuum expectation value. Following the general choices in the analysis of dark matter searches in literatures, we take $g_{\mathrm{DM}}^{S}=g_{q}^{S}=1$ and $g_{\mathrm{DM}}^{A}=$ $1, g_{q}^{A}=\frac{1}{4}$ in the calculations below. Under the above assumptions the dark matter models are described by two parameters, i.e. the dark matter mass $m_{\chi}$ and the mediator mass $m_{S}$ or $m_{V}$. The scan range for these parameters is

$$
5 \mathrm{GeV}<m_{\chi}, m_{S}, m_{V}<10 \mathrm{TeV}
$$

Induced by the interactions in eqs. (3.1) and (3.2), the pairs of dark matter particle $\chi$ can either annihilate into SM quark pairs via the mediator particle in s channel $\bar{\chi} \chi \rightarrow$ $S / V \rightarrow \bar{q} q$, or annihilate into the mediator pairs in t channel followed by mediators decaying to SM quarks $\bar{\chi} \chi \rightarrow S S / V V \rightarrow \bar{q} q \bar{q}^{\prime} q^{\prime}$. The resulting cosmic ray spectra can thus be categorized into 2-body spectrum and 4-body spectrum, respectively.

The dark matter source term contributing to the cosmic ray species $i$ is given by

$$
Q_{i}^{\chi}(r, p)=\frac{\rho_{\chi}^{2}(r)\left\langle\sigma_{\text {ann }} v\right\rangle}{2 m_{\chi}^{2}} \frac{d N_{i}}{d E}
$$

where $\left\langle\sigma_{\text {ann }} v\right\rangle$ is the total velocity averaged dark matter annihilation cross section of all kinematically allowed channels. $d N_{i} / d E$ is the total energy spectrum of cosmic ray particle $i$ produced in the annihilation, that is the sum of 2-body spectrum and 4-body spectrum $d N_{i} / d E=\left(d N_{i} / d E\right)_{2-\text { body }}+\left(d N_{i} / d E\right)_{4-\text { body }}$. 
For the 2-body spectrum, one has

$$
\left(\frac{d N_{i}}{d E}\right)_{2-\text { body }}=\sum_{q} \frac{\left\langle\sigma_{\mathrm{ann}} v\right\rangle_{q}}{\left\langle\sigma_{\mathrm{ann}} v\right\rangle} \frac{d N_{i}^{q}}{d E}+\frac{\left\langle\sigma_{\mathrm{ann}} v\right\rangle_{g}}{\left\langle\sigma_{\mathrm{ann}} v\right\rangle} \frac{d N_{i}^{g}}{d E}
$$

with $\left\langle\sigma_{\text {ann }} v\right\rangle_{q}=\sigma_{\text {ann }} v(\bar{\chi} \chi \rightarrow S / V \rightarrow q \bar{q}),\left\langle\sigma_{\text {ann }} v\right\rangle_{g}=\sigma_{\text {ann }} v(\bar{\chi} \chi \rightarrow S \rightarrow g g)$ for the pseudoscalar mediator case and $\left\langle\sigma_{\text {ann }} v\right\rangle_{g}=0$ for the axialvector mediator case. $d N_{i}^{q} / d E$ and $d N_{i}^{g} / d E$ are the cosmic ray spectra given by dark matter direct annihilating into quark pairs $\bar{q} q$ and gluons $g g$, respectively. The 4-body spectrum is

$$
\left(\frac{d N_{i}}{d E}\right)_{4-\text { body }}=\sum_{q} \frac{\left\langle\sigma_{\text {ann }} v\right\rangle_{\mathrm{Med}}}{\left\langle\sigma_{\text {ann }} v\right\rangle} \frac{\Gamma_{\mathrm{Med} \rightarrow q \bar{q}}}{\Gamma_{\mathrm{Med}}} \frac{d \bar{N}_{i}^{q}}{d E}+\frac{\left\langle\sigma_{\mathrm{ann}} v\right\rangle_{\mathrm{Med}}}{\left\langle\sigma_{\mathrm{ann}} v\right\rangle} \frac{\Gamma_{\mathrm{Med} \rightarrow g g}}{\Gamma_{\mathrm{Med}}} \frac{d \bar{N}_{i}^{g}}{d E},
$$

where $\left\langle\sigma_{\mathrm{ann}} v\right\rangle_{\mathrm{Med}}=\sigma_{\mathrm{ann}} v(\bar{\chi} \chi \rightarrow S S / V V), \Gamma_{\mathrm{Med} \rightarrow q \bar{q}}=\Gamma_{S / V \rightarrow q \bar{q}}$ and the total decay width of the mediator is $\Gamma_{\text {Med }}=\Gamma_{S / V}$. $\Gamma_{\text {Med } \rightarrow g g}=\Gamma_{S \rightarrow g g}$ for the pseudoscalar mediator case and $\Gamma_{\text {Med } \rightarrow g g}=0$ for the axialvector mediator case. $d \bar{N}_{i}^{q} / d E$ and $d \bar{N}_{i}^{g} / d E$ are the cosmic ray spectra in the lab frame given by the spectrum from the mediator decay in its rest frame, denoted by $d N_{i}^{q} / d E_{0}$ and $d N_{i}^{g} / d E_{0}$, after a Lorentz boost [28, 29]:

$$
\frac{d \bar{N}_{i}^{q, g}}{d E}=2 \int_{t_{1, \min }}^{t_{1, \max }} \frac{d x_{0}}{x_{0} \sqrt{1-\epsilon^{2}}} \frac{d N_{i}^{q, g}}{d E_{0}}
$$

where

$$
\begin{aligned}
& t_{1, \max }=\min \left[1, \frac{2 x}{\epsilon^{2}}\left(1+\sqrt{1-\epsilon^{2}}\right)\right], \\
& t_{1, \text { min }}=\frac{2 x}{\epsilon^{2}}\left(1-\sqrt{1-\epsilon^{2}}\right)
\end{aligned}
$$

with $\epsilon=m_{\mathrm{Med}} / m_{\chi}$ and $x=E / m_{\chi} \leq 0.5$. The expressions of dark matter annihilation cross sections and mediator decay widths in eqs. (3.5) and (3.6) are collected in appendix. As a result of the non-trivial involvement of the mediator, $\left\langle\sigma_{\text {ann }} v\right\rangle$ and $d N_{i} / d E$ are dependent on both the dark matter mass and the mediator mass. AMS-02 data thus play an important role in constraining these two parameters.

We show the $\left\langle\sigma_{\mathrm{ann}} v\right\rangle_{i} /\left\langle\sigma_{\mathrm{ann}} v\right\rangle$ as a function of $m_{\chi}$ in figure 1. For pseudoscalar mediator case, we find that $\bar{\chi} \chi \rightarrow g g$ channel is dominant in small dark matter mass region. After $t \bar{t}$ channel is open, $\bar{\chi} \chi \rightarrow \bar{q} q$ channel turns to be dominant. $\bar{\chi} \chi \rightarrow S S$ channel is always very small as it is a process through $\mathrm{p}$ wave. For axialvector mediator case, $\bar{\chi} \chi \rightarrow \bar{q} q$ is dominant before $\bar{\chi} \chi \rightarrow V V$ is forbidden and after $\bar{\chi} \chi \rightarrow \bar{t} t$ is open. In figure 2 we show the total antiproton spectrum $x^{2} d N_{i} / d E$ as a function of $x=E / m_{\chi}$.

We use a generalized Navarro-Frenk-White (NFW) profile to describe dark matter spatial distribution $[30,31]$

$$
\rho_{\chi}(r)=\rho_{0} \frac{\left(r / r_{s}\right)^{-\gamma}}{\left(1+r / r_{s}\right)^{3-\gamma}} .
$$

The NFW profile is a traditional benchmark choice motivated by N-body simulations. Here the coefficient $\rho_{0}$ is $0.26 \mathrm{GeV} / \mathrm{cm}^{3}$ and the radius of the galactic diffusion disk is $r_{s}=20$ 

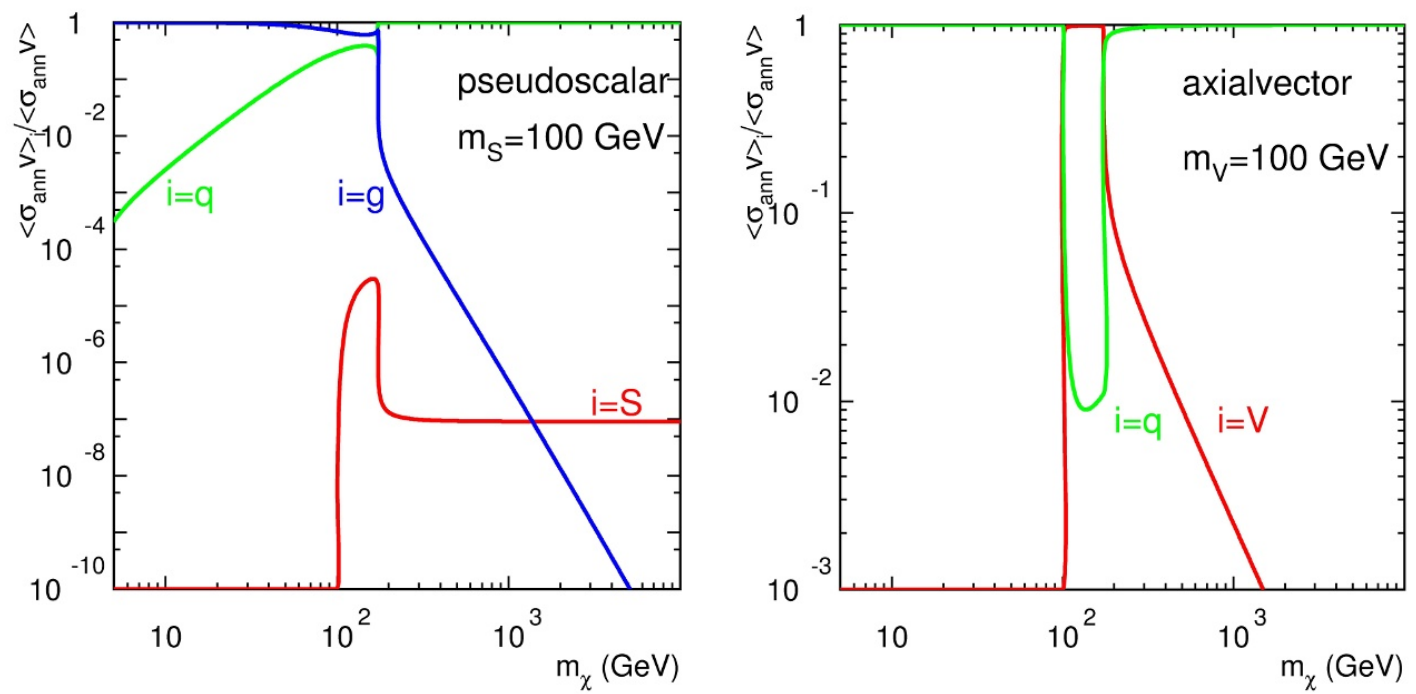

Figure 1. The annihilation cross section fractions $\left\langle\sigma_{\mathrm{ann}} v\right\rangle_{i} /\left\langle\sigma_{\mathrm{ann}} v\right\rangle$ as a function of $m_{\chi}$ for the pseudoscalar mediator case (left) and the axialvector mediator case (right). The mediator mass is fixed to be $100 \mathrm{GeV}$.
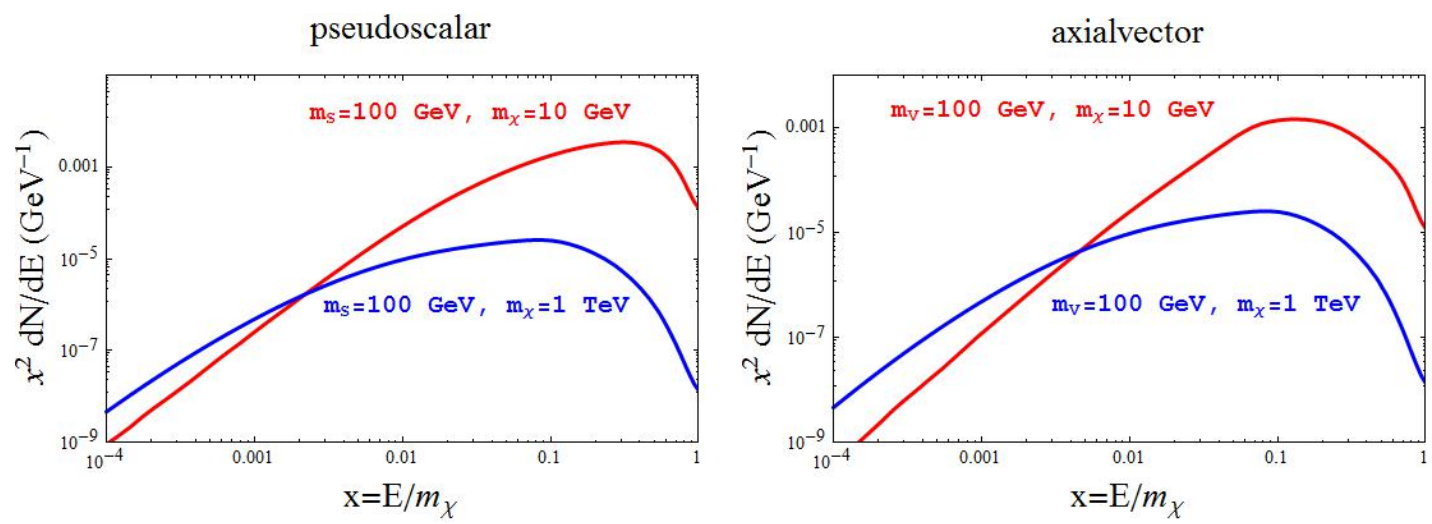

Figure 2. Total antiproton spectrum $x^{2} d N_{i} / d E$ as a function of $x=E / m_{\chi}$ for the pseudoscalar mediator case (left) and the axialvector mediator case (right).

kpc. We fix the inner slope of the halo profile as $\gamma=1$. Note that the Einasto profile is one of the two most commonly used dark matter density profiles [8], together with NFW profile. However, the shape parameter in Einasto varies from simulation to simulation. In order to keep minimal parameter dependence, we adopt NFW profile only.

\section{Results}

As discussed in section 2, the propagation and injection parameters of cosmic rays are determined by fitting the $\mathrm{B} / \mathrm{C}$ and ${ }^{10} \mathrm{Be} /{ }^{9} \mathrm{Be}$ data and the proton data from AMS-02, respectively. The parameters in table 1 thus imply the prediction of cosmic ray measurements inferred from standard astrophysical sources. One can investigate the constraint on extra sources, such as dark matter, based on this fiducial astrophysical background. 
For each group of dark matter mass and mediator mass, we use PPPC4DMID [32] to generate the antiproton spectrum in eqs. (3.5) and (3.6), and calculate the dark matter annihilation cross sections following the formulas in appendix. These dark matter model dependent variables are then passed into the public code Galprop v54 [33-37] to ensure that near Earth cosmic ray fluxes from dark matter annihilation and background spectra are obtained in a consistent way.

The resultant cosmic ray fluxes, together with the measured data points, are put into a composite likelihood function, defined as

$$
-2 \ln \mathcal{L}=\sum_{i} \frac{\left(f_{i}^{\text {th }}-f_{i}^{\text {exp }}\right)^{2}}{\sigma_{i}^{2}} .
$$

Here $f_{i}^{\text {th }}$ are the theoretical predictions and $f_{i}^{\text {exp }}$ are the corresponding central value of the experimental data. The uncertainty $\sigma_{i}$ combines the theoretical and experimental uncertainties in quadrature. We stipulate a $50 \%$ uncertainty of the theoretical prediction of antiproton flux and antiproton-to-proton ratio according to the estimates in refs. [6, 3840]. This uncertainty takes into account, amongst other, the uncertainty related to the fixed propagation parameters. The sum in eq. (4.1) runs over all the AMS-02 antiproton cosmic ray spectral data points: the antiproton flux (57 points) and antiproton-proton ratio (57 points). Note that the antiproton flux and antiproton-proton ratio data are not fully uncorrelated. However, the systematic uncertainties are different on the two data sets [2], such as the uncertainties in the event selection, detector materials, etc. These uncertainties are added together to give the systematic errors for the two data sets individually in the AMS-02 analysis. Thus, in order to make a comprehensive study, we include the central values and uncertainties of the two data sets in our likelihood function.

As the dark matter-nucleon elastic scattering cross sections induced by the simplified models we consider are spin-dependent, the most stringent constraints come from collider search and indirect detection of dark matter [41-44]. LHC performed dark matter search using events with large missing transverse momentum plus energetic jets [41] and dijet events $[42,43]$ at $13 \mathrm{TeV}$ collisions. Their exclusion limits can be directly presented in the plane of dark matter mass vs. mediator mass for simplified model with a pseudoscalar mediator or an axialvector mediator. Moreover, Fermi Large Area Telescope (LAT) searched for gamma ray emission from Milky Way satellite galaxies using 6 years of data. They recently released the observed constraints on the dark matter annihilation cross section for pure $b \bar{b}$ channel [45]. We can convert the Fermi-LAT limit into a bound on our dark matter annihilation cross section. Suppose that the $b \bar{b}$ component of the total annihilation cross section fixed by dark matter mass and mediator mass satisfies

$$
\left\langle\sigma_{\mathrm{ann}} v\right\rangle>\langle\sigma v\rangle_{b b}^{\text {Fermi-limit }} \frac{\left\langle\sigma_{\mathrm{ann}} v\right\rangle}{\left\langle\sigma_{\mathrm{ann}} v\right\rangle_{b}}
$$

we claim the corresponding set of $m_{\chi}, m_{\mathrm{Med}}$ is excluded.

Figures 3 and 4 show our main results: AMS-02 cosmic ray flux data are consistent with the dark matter framework within the uncertainties. The two plots in each figure 

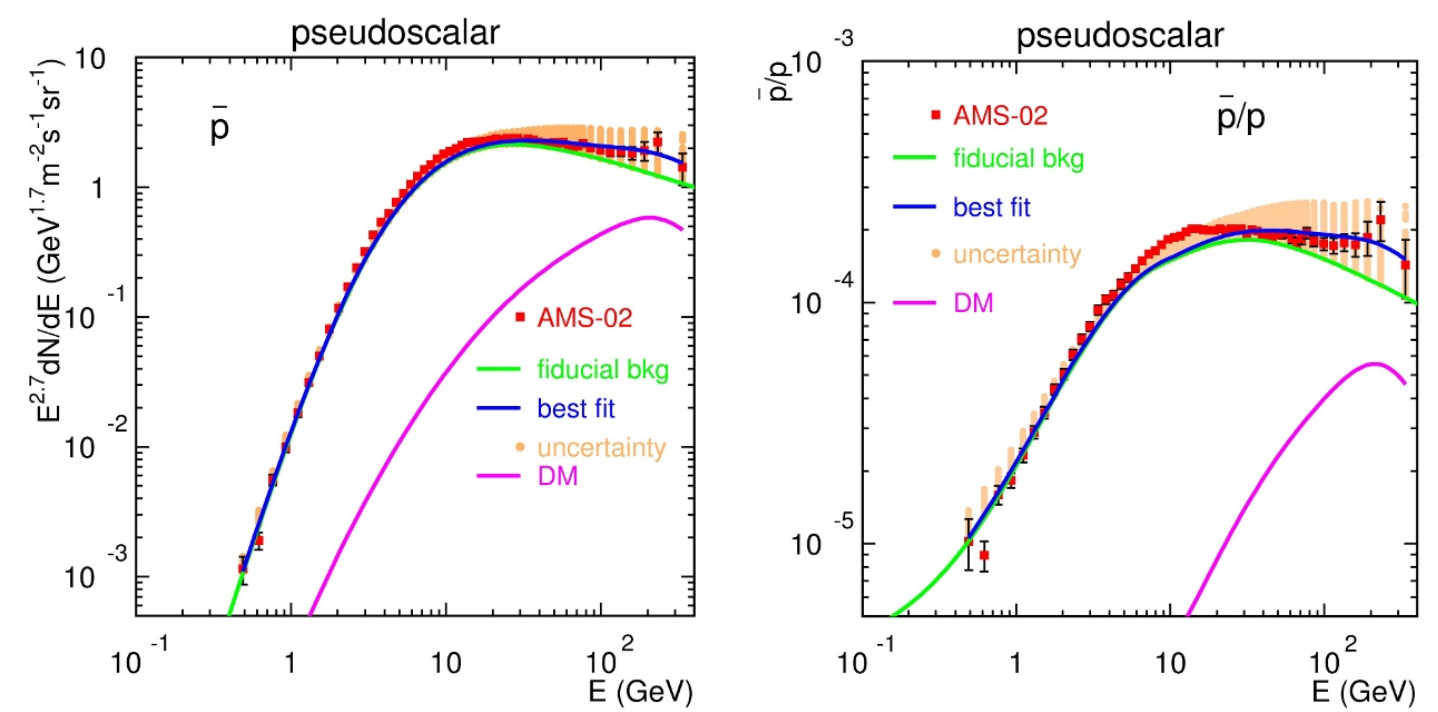

Figure 3. Antiproton flux (left) and antiproton-to-proton ratio (right) observed by AMS-02 (red dots and dark error bars) in the simplified dark matter model with a pseudoscalar mediator. The blue solid line shows the prediction of the total cosmic ray flux with dark matter parameter values that best fit the AMS-02 data. The total predicted flux is the sum of the background flux (green curve) and the dark matter contribution (purple curve). Salmon dots indicate the $2 \sigma$ confidence region of the prediction.

display the antiproton cosmic ray: antiproton flux and antiproton-to-proton ratio. AMS02 central value measurements are shown by red dots and error bars in black indicate measurement uncertainties. The green solid curves are obtained using the parameters shown in table 1 and display the predicted background flux originating from standard astrophysical sources. The blue solid lines show the predictions of the total cosmic ray flux with dark matter contribution that fit the AMS-02 data best and are the sum of the background flux (green curve) and the dark matter contribution at the best fit point (purple curve). As varying the two mass parameters in simplified dark matter models and thus the likelihood function, we can also obtain the confidence regions of dark matter model parameters. We calculate $1(2) \sigma$ confidence region by increasing the likelihood function from its best fit value, whilst scanning the two mass parameters, until $-2 \ln \mathcal{L}$ changes by 2.30 (6.18). The dark matter contributions to the observables are then calculated using the dark matter model parameters in the $2 \sigma$ confidence region. These contributions are added to the background flux and give the theoretical uncertainty of the dark matter prediction (salmon colored vertical bars). The plots show that adding a dark matter contribution to the background flux yields a better fit to the AMS-02 data.

In the left frame of figure 5 we show the regions of the mass parameter space preferred by the AMS-02 data and the LHC limit for the pseudoscalar mediator case. Solid circles and squares denote the estimated $1 \sigma$ and $2 \sigma$ confidence regions, respectively. We find that the AMS-02 antiproton data favor $700 \mathrm{GeV} \lesssim m_{\chi} \lesssim 5 \mathrm{TeV}$ region at about $1 \sigma$ confidence level. The LHC excludes a part of the $2 \sigma$ confidence region with $m_{\chi} \lesssim 170 \mathrm{GeV}$ and $300 \mathrm{GeV} \lesssim m_{S} \lesssim 420 \mathrm{GeV}$. 

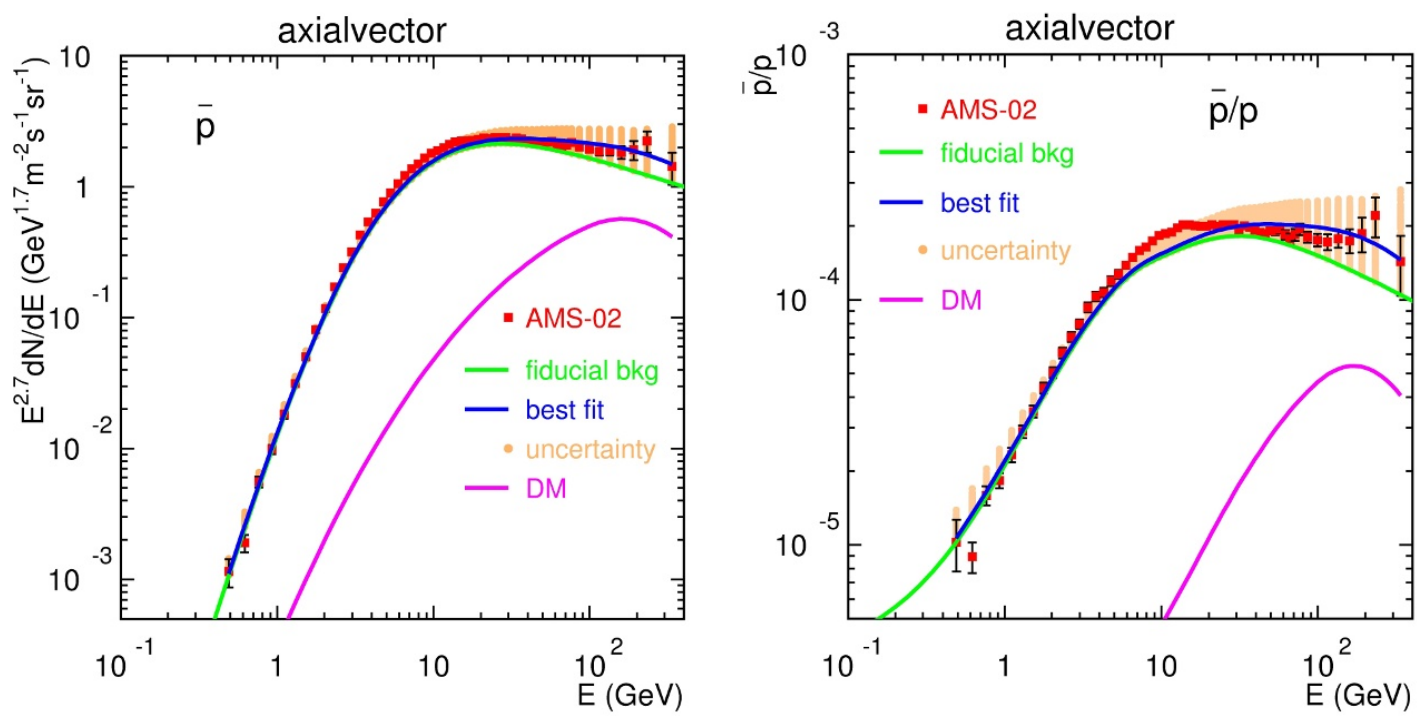

Figure 4. Antiproton flux (left) and antiproton-to-proton ratio (right) observed by AMS-02 (red dots and dark error bars) in the simplified dark matter model with an axialvector mediator.

The right frame of figure 5 shows that the AMS-02 data require an effective dark matter annihilation cross section in the region of $1 \times 10^{-25}-1 \times 10^{-24}\left(5 \times 10^{-27}-2 \times 10^{-24}\right) \mathrm{cm}^{3} / \mathrm{s}$ at about 1(2) $\sigma$ C.L. The LHC excludes a part of the region below thermal relic cross section, denoted by green dots. After $t \bar{t}$ channel is open, it becomes the dominant annihilation channel and the $b \bar{b}$ component is highly suppressed. The rescale factor $\frac{\left\langle\sigma_{\text {ann }} v\right\rangle}{\left\langle\sigma_{\text {ann }} v\right\rangle_{b}}$ in eq. (4.2) is thus largely enhanced, so is the rescaled Fermi-LAT bound $\langle\sigma v\rangle_{b b}^{\text {Fermi-limit }} \frac{\left\langle\sigma_{\text {ann }} v\right\rangle}{\left\langle\sigma_{\text {ann }} v\right\rangle_{b}}$. The Fermi-LAT bound thus becomes rather weak after $t \bar{t}$ channel is open and does not constrain the AMS-02 favored region.

In the left frame of figure 6 , for the axialvector mediator case, we can see that the AMS02 antiproton data favor the region with $m_{\chi} \gtrsim 700 \mathrm{GeV}$ and $200 \mathrm{GeV} \lesssim m_{V} \lesssim 1 \mathrm{TeV}$ at about $1 \sigma$ confidence level. The region with $m_{\chi} \gtrsim 1 \mathrm{TeV}$ and $m_{V} \lesssim 500 \mathrm{GeV}$ can evade the LHC limit.

The dark matter annihilation with axialvector mediator requires the cross section in the region of $1 \times 10^{-25}-4 \times 10^{-24}\left(1 \times 10^{-26}-4 \times 10^{-24}\right) \mathrm{cm}^{3} / \mathrm{s}$ at about $1(2) \sigma$ C.L. as shown in the right plot of figure 6 . The LHC excludes a majority of the region below $3 \times 10^{-25} \mathrm{~cm}^{3} / \mathrm{s}$, denoted by green dots. The Fermi-LAT bound does not constrain the AMS-02 favored region either.

In order to investigate the impact of different dark matter halo profile, we examine the truncated Isothermal profile [47] for the pseudoscalar mediator framework as an example

$$
\rho_{\chi}(r)=\frac{\rho_{0}}{1+\left(r / r_{s}\right)^{2}},
$$

where the coefficient $\rho_{0}$ is $1.65 \mathrm{GeV} / \mathrm{cm}^{3}$ and $r_{s}=4 \mathrm{kpc}$. As shown in figure 7 , we find that including the dark matter contribution to the background flux fits the data well in this case. In addition, the AMS-02 favored region of masses agrees with that using NFW profile. 

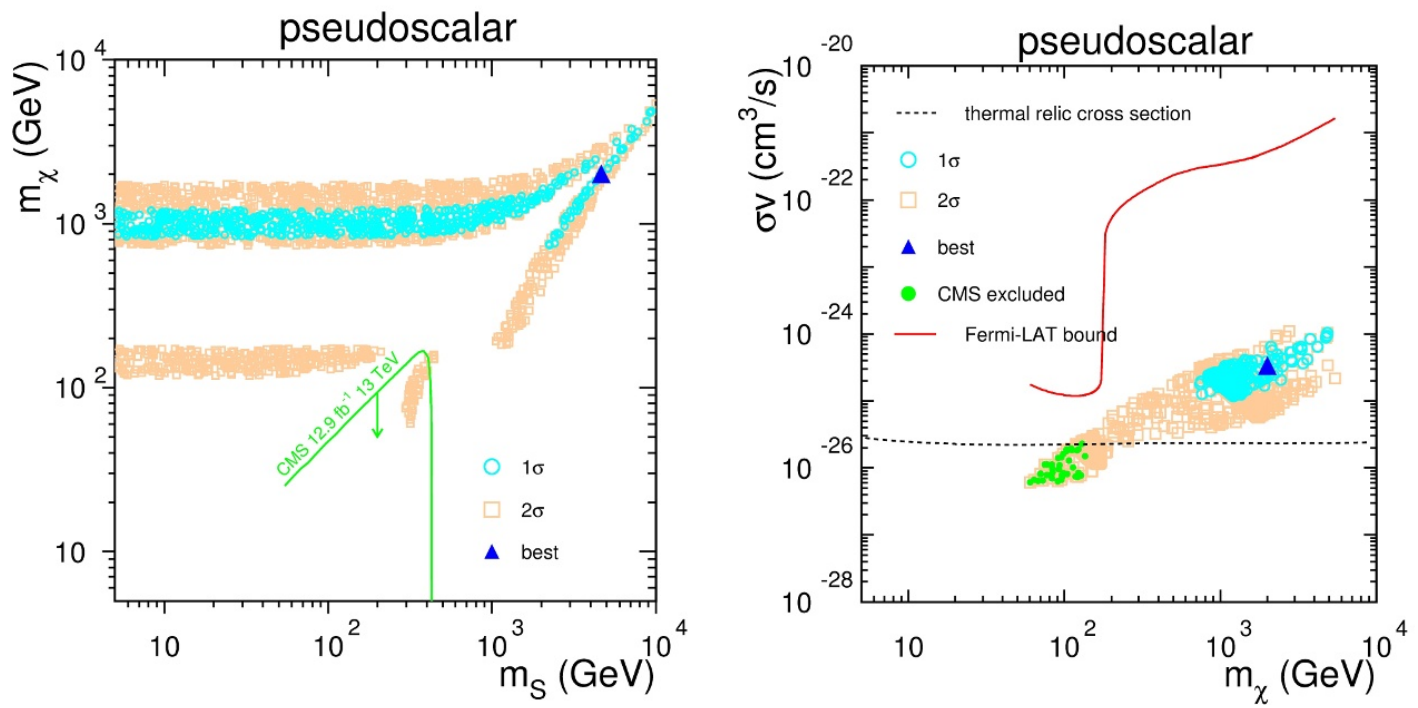

Figure 5. Left: the AMS-02 favored region of masses $\left(m_{\chi}\right.$ vs. $\left.m_{S}\right)$ in the simplified dark matter model with a pseudoscalar mediator we consider. The solid circles and squares estimate $1 \sigma$ and $2 \sigma$ confidence regions, respectively. The best fit point is indicated by a triangle. The green curve is the LHC exclusion limit [41]. Right: the AMS-02 favored region of cross sections ( $\sigma v$ vs. $m_{\chi}$ ). The green points are excluded by LHC search. The red curve is the converted upper bound from Fermi-LAT, i.e. the right hand side of eq. (4.2). The black dashed curve corresponds to the thermal cross section [46].
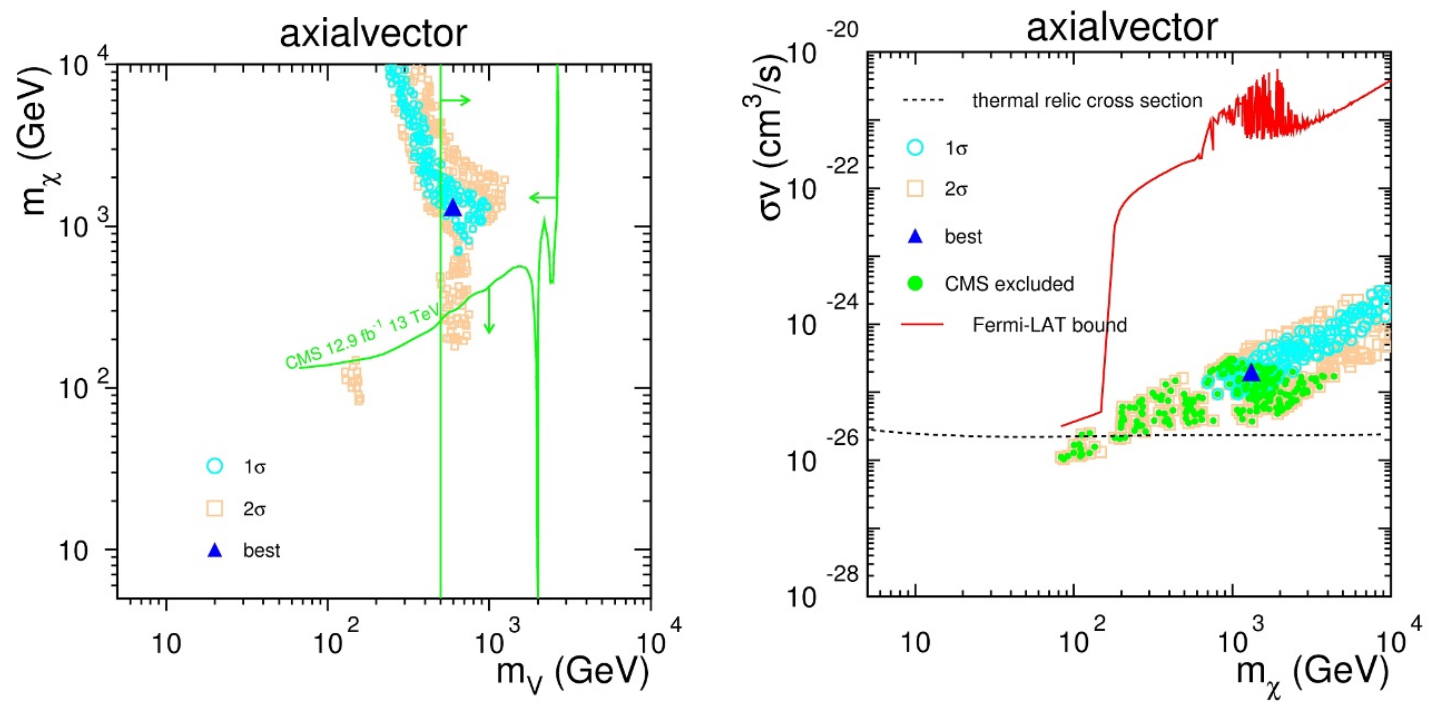

Figure 6. Left: the AMS-02 favored region of masses $\left(m_{\chi}\right.$ vs. $\left.m_{V}\right)$ in the simplified dark matter model with an axialvector mediator we consider. The LHC exclusion limits are from refs. [41, 42] and [43]. Right: the AMS-02 favored region of cross sections ( $\sigma v$ vs. $m_{\chi}$ ). 

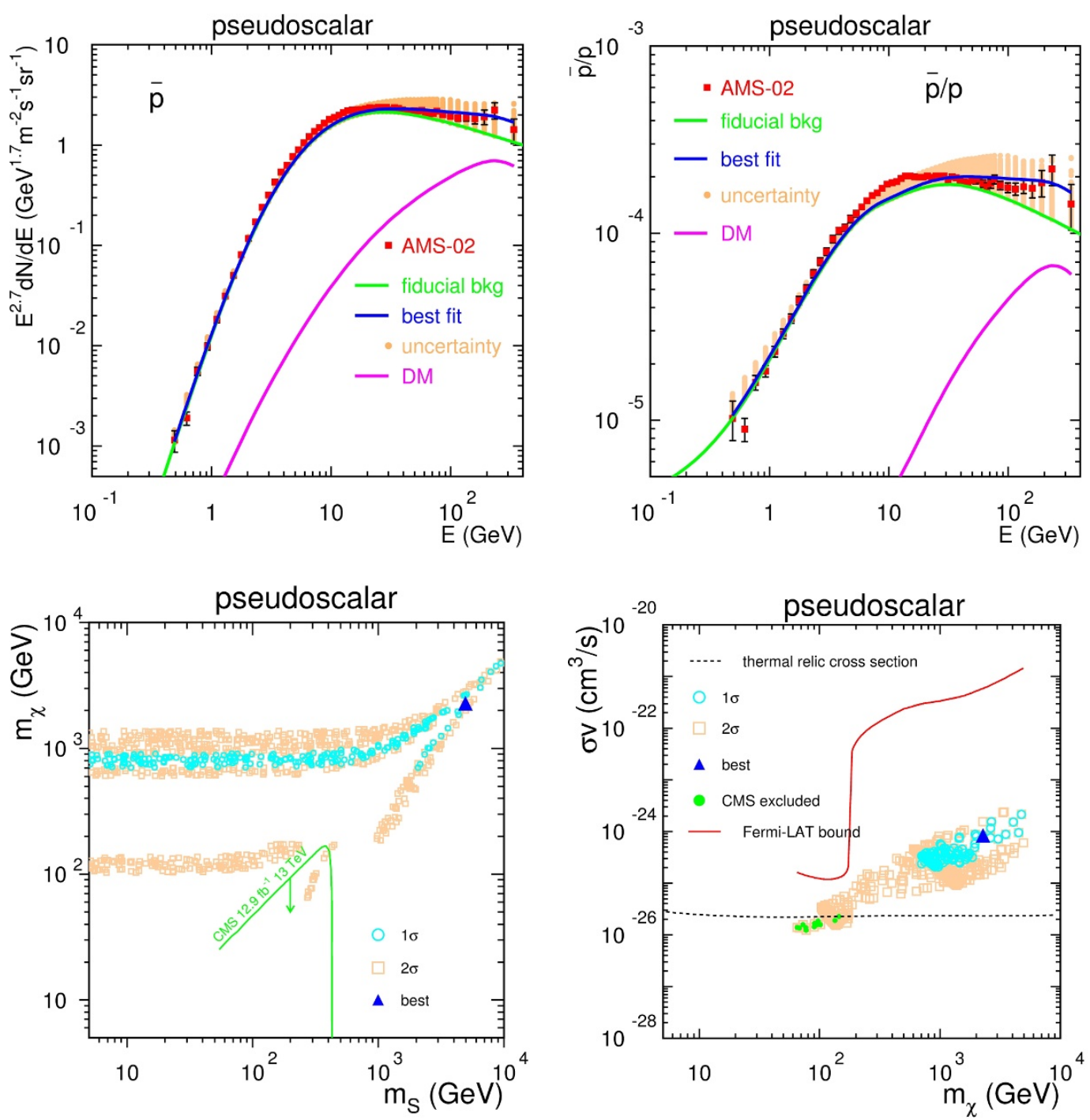

Figure 7. Antiproton flux (top left), antiproton-to-proton ratio (top right), the AMS-02 favored region of masses (bottom left) and annihilation cross section (bottom right) in the pseudoscalar mediator model with the truncated Isothermal profile.

The AMS-02 analysis of antiproton flux and antiproton-to-proton ratio data sets was based on the same antiproton events. Although the antiproton-to-proton ratio data reply on proton events in addition and their systematic uncertainties are different, the two data sets might be correlated. In order to check the possible correlation influence, we investigate the case with only the antiproton flux data points being included in the likelihood in eq. (4.1), for the pseudoscalar mediator framework as an example. In this case we find the best fit mass parameters are $m_{\chi}=1.75 \mathrm{TeV}$ and $m_{S}=4.16 \mathrm{TeV}$ which are close to the results from including both of the two data sets in the likelihood. After we extract the best fit dark matter model parameters, we calculate the antiproton-to-proton ratio using the best fit parameters. As shown in figure 8, the antiproton-to-proton ratio data and the best fit (obtained without this data) agree well. This is an important cross check of the internal consistency of the dark matter framework and our parameter extraction procedure. 

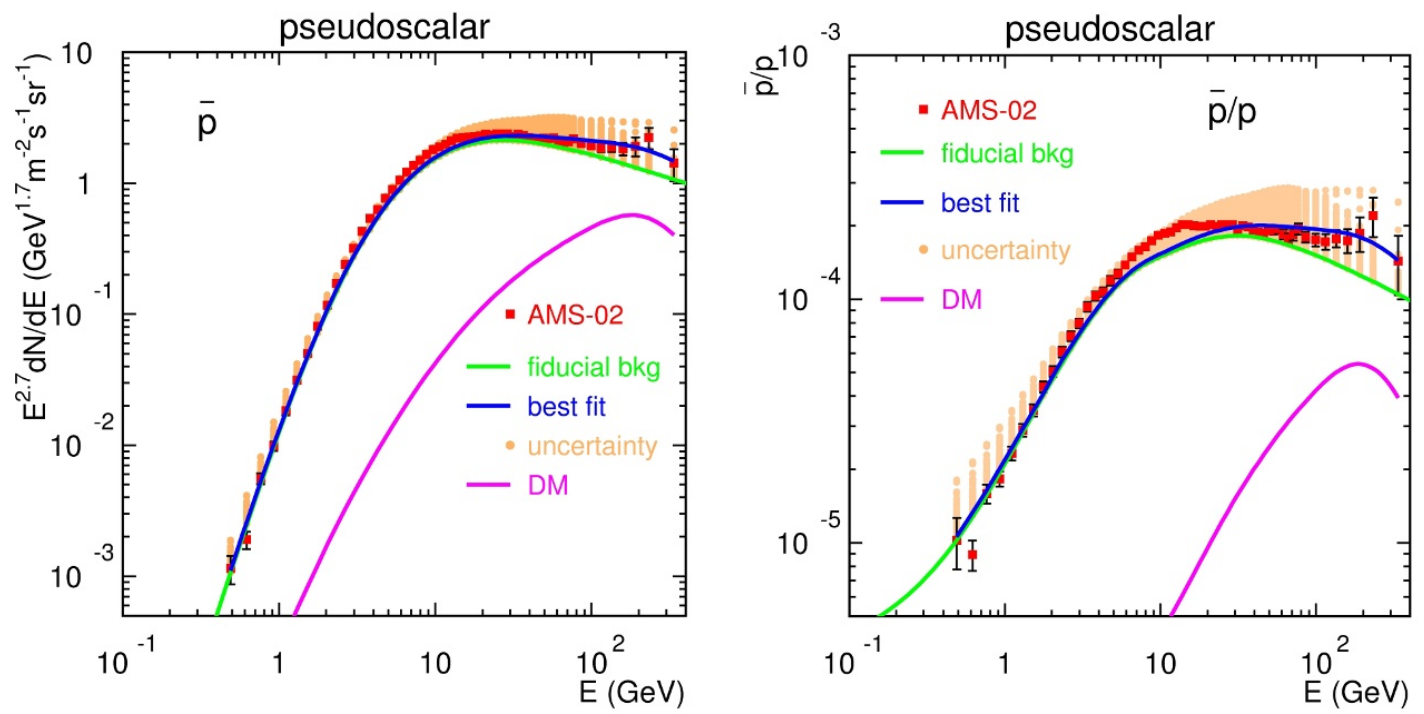

Figure 8. Antiproton flux (left) and antiproton-to-proton ratio (right) in the pseudoscalar mediator model with only the antiproton flux data points included in the likelihood in eq. (4.1).

\section{Conclusions}

In this work we examine the plausibility of leptophobic dark matter annihilation contributing to the recent AMS-02 data, i.e. the antiproton flux and antiproton-to-proton ratio. Besides the standard astrophysical cosmic ray flux prediction we include a dark matter component. Our choice of the dark matter model is two simplified models of a Dirac fermionic dark matter, with leptophobic pseudoscalar and axialvector mediators that couple only to SM quarks and dark matter particles. The fluxes from standard astrophysical sources and dark matter annihilation are propagated through the Galaxy using the same set of diffusion parameters. The propagation and injection parameters are determined by fitting the latest AMS-02 cosmic ray fluxes of nuclei.

We have shown that not only AMS-02 observations are consistent with the dark matter framework within the uncertainties, but also including a dark matter contribution to the background flux gives a better fit to the data. We also estimated the most plausible parameter regions of the dark matter parameter space in light of AMS-02 data. The observation of antiproton prefers a dark matter (mediator) mass in the $700 \mathrm{GeV}-5 \mathrm{TeV}$ ( $5 \mathrm{GeV}-$ $10 \mathrm{TeV}$ ) region for the annihilation with pseudoscalar mediator and in the $700 \mathrm{GeV}-10 \mathrm{TeV}$ $(200 \mathrm{GeV}-1 \mathrm{TeV})$ region for the annihilation with axialvector mediator, respectively, at about $68 \%$ confidence level. The AMS-02 data require an effective dark matter annihilation cross section in the region of $1 \times 10^{-25}-1 \times 10^{-24}\left(1 \times 10^{-25}-4 \times 10^{-24}\right) \mathrm{cm}^{3} / \mathrm{s}$ for the simplified model with pseudoscalar (axialvector) mediator. The LHC excludes a part of the region below thermal relic cross section for the pseudoscalar mediator model and the region with axialvector mediator mass greater than $500 \mathrm{GeV}$. The Fermi-LAT bound does not constrain the AMS-02 favored region. 


\section{Acknowledgments}

We would like to thank Csaba Balázs and Thomas Jacques for discussions. We also thank Qiang Yuan for helping with Galprop. The National Computational Infrastructure (NCI), the Southern Hemisphere's fastest supercomputer, is also gratefully acknowledged.

\section{A Expressions of mediator decay widths and dark matter annihilation cross sections}

The mediator decay widths for the pseudoscalar mediator case [17]:

$$
\begin{aligned}
\Gamma_{S \rightarrow \bar{\chi} \chi} & =\frac{\left(g_{\mathrm{DM}}^{S}\right)^{2} m_{S}}{8 \pi}\left(1-\frac{4 m_{\chi}^{2}}{m_{S}^{2}}\right)^{1 / 2}, \\
\Gamma_{S \rightarrow \bar{q} q} & =N_{c} \frac{\left(g_{q}^{S}\right)^{2} m_{S}}{8 \pi} \frac{m_{q}^{2}}{v_{0}^{2}}\left(1-\frac{4 m_{q}^{2}}{m_{S}^{2}}\right)^{1 / 2} q=u, d, s, c, b, t, \\
\Gamma_{S \rightarrow g g} & =\frac{\left(g_{q}^{S}\right)^{2} \alpha_{s}^{2}\left(m_{S}\right) m_{S}^{3}}{32 \pi^{3} v_{0}^{2}}\left|\frac{4 m_{t}^{2}}{m_{S}^{2}} \arctan ^{2}\left(\left(\frac{4 m_{t}^{2}}{m_{S}^{2}}-1\right)^{-1 / 2}\right)\right|^{2}, \\
\Gamma_{S} & =\Gamma_{S \rightarrow \bar{\chi} \chi}+\Gamma_{S \rightarrow \bar{q} q}+\Gamma_{S \rightarrow g g}
\end{aligned}
$$

The dark matter annihilation cross sections for the pseudoscalar mediator case [14]:

$$
\begin{aligned}
& \sigma_{\mathrm{ann}} v(\bar{\chi} \chi \rightarrow S \rightarrow \bar{q} q)=\frac{\left(g_{\mathrm{DM}}^{S}\right)^{2}\left(g_{q}^{S}\right)^{2} N_{c}}{\left(4 m_{\chi}^{2}-m_{S}^{2}\right)^{2}+m_{S}^{2} \Gamma_{S}^{2}} \frac{m_{\chi}^{2}}{2 \pi} \frac{m_{q}^{2}}{v_{0}^{2}}\left(1-\frac{m_{q}^{2}}{m_{\chi}^{2}}\right)^{1 / 2}, \\
& \sigma_{\mathrm{ann}} v(\bar{\chi} \chi \rightarrow S \rightarrow g g)=\frac{\left(g_{\mathrm{DM}}^{S}\right)^{2}\left(g_{q}^{S}\right)^{2}}{\left(4 m_{\chi}^{2}-m_{S}^{2}\right)^{2}+m_{S}^{2} \Gamma_{S}^{2}} \frac{\alpha_{s}^{2}\left(2 m_{\chi}\right) m_{t}^{4}}{2 \pi^{3} v_{0}^{2}} \\
& \times\left|\frac{m_{t}^{2}}{m_{\chi}^{2}} \arctan ^{2}\left(\left(\frac{m_{t}^{2}}{m_{\chi}^{2}}-1\right)^{-1 / 2}\right)\right|^{2}, \\
& \sigma_{\mathrm{ann}} v(\bar{\chi} \chi \rightarrow S S)=\left(g_{\mathrm{DM}}^{S}\right)^{4} \frac{m_{\chi}^{2}\left(m_{\chi}^{4}-2 m_{\chi}^{2} m_{S}^{2}+m_{S}^{4}\right)}{24 \pi\left(2 m_{\chi}^{2}-m_{S}^{2}\right)^{4}}\left(1-\frac{m_{S}^{2}}{m_{\chi}^{2}}\right)^{1 / 2} v^{2},
\end{aligned}
$$

where $v \simeq 10^{-3}$.

The mediator decay widths for the axialvector mediator case [15]:

$$
\begin{aligned}
\Gamma_{V \rightarrow \bar{\chi} \chi} & =\frac{\left(g_{\mathrm{DM}}^{A}\right)^{2} m_{V}}{12 \pi}\left(1-\frac{4 m_{\chi}^{2}}{m_{V}^{2}}\right)^{3 / 2}, \\
\Gamma_{V \rightarrow \bar{q} q} & =\frac{\left(g_{q}^{A}\right)^{2} m_{V}}{4 \pi}\left(1-\frac{4 m_{q}^{2}}{m_{V}^{2}}\right)^{3 / 2} q=u, d, s, c, b, t, \\
\Gamma_{V} & =\Gamma_{V \rightarrow \bar{\chi} \chi}+\Gamma_{V \rightarrow \bar{q} q}
\end{aligned}
$$


The dark matter annihilation cross sections for the axialvector mediator case [15]:

$$
\begin{aligned}
\sigma_{\mathrm{ann}} v(\bar{\chi} \chi \rightarrow V \rightarrow \bar{q} q) & =\frac{\left(g_{\mathrm{DM}}^{A}\right)^{2}\left(g_{q}^{A}\right)^{2}}{\left(4 m_{\chi}^{2}-m_{V}^{2}\right)^{2}+m_{V}^{2} \Gamma_{V}^{2}}\left(1-\frac{m_{q}^{2}}{m_{\chi}^{2}}\right)^{1 / 2} \frac{3 m_{q}^{2}\left(4 m_{\chi}^{2}-m_{V}^{2}\right)^{2}}{2 \pi m_{V}^{4}} \\
\sigma_{\mathrm{ann}} v(\bar{\chi} \chi \rightarrow V V) & =\frac{\left(g_{\mathrm{DM}}^{A}\right)^{4}}{4 \pi m_{\chi}\left(2 m_{\chi}^{2}-m_{V}^{2}\right)^{2}}\left(m_{\chi}^{2}-m_{V}^{2}\right)^{3 / 2}
\end{aligned}
$$

Open Access. This article is distributed under the terms of the Creative Commons Attribution License (CC-BY 4.0), which permits any use, distribution and reproduction in any medium, provided the original author(s) and source are credited.

\section{References}

[1] AMS collaboration, M. Aguilar et al., Precision measurement of the proton flux in primary cosmic rays from rigidity 1 GV to 1.8 TV with the Alpha Magnetic Spectrometer on the International Space Station, Phys. Rev. Lett. 114 (2015) 171103 [INSPIRE].

[2] AMS collaboration, M. Aguilar et al., Antiproton flux, antiproton-to-proton flux ratio and properties of elementary particle fluxes in primary cosmic rays measured with the Alpha Magnetic Spectrometer on the International Space Station, Phys. Rev. Lett. 117 (2016) 091103 [INSPIRE].

[3] AMS collaboration, M. Aguilar et al., Precision measurement of the helium flux in primary cosmic rays of rigidities 1.9 GV to 3 TV with the Alpha Magnetic Spectrometer on the International Space Station, Phys. Rev. Lett. 115 (2015) 211101 [INSPIRE].

[4] M. Stref and J. Lavalle, Modeling dark matter subhalos in a constrained galaxy: global mass and boosted annihilation profiles, Phys. Rev. D 95 (2017) 063003 [arXiv:1610.02233] [INSPIRE].

[5] A. Cuoco, M. Krämer and M. Korsmeier, Novel dark matter constraints from antiprotons in the light of AMS-02, arXiv:1610.03071 [INSPIRE].

[6] M.-Y. Cui, Q. Yuan, Y.-L.S. Tsai and Y.-Z. Fan, A possible dark matter annihilation signal in the AMS-02 antiproton data, arXiv:1610.03840 [INSPIRE].

[7] J. Feng, N. Tomassetti and A. Oliva, Bayesian analysis of spatial-dependent cosmic-ray propagation: astrophysical background of antiprotons and positrons, Phys. Rev. D 94 (2016) 123007 [arXiv: 1610.06182] [INSPIRE].

[8] X.-J. Huang, C.-C. Wei, Y.-L. Wu, W.-H. Zhang and Y.-F. Zhou, Antiprotons from dark matter annihilation through light mediators and a possible excess in AMS-02 $\bar{p} / p$ data, Phys. Rev. D 95 (2017) 063021 [arXiv:1611.01983] [INSPIRE].

[9] W. Liu, X.-J. Bi, S.-J. Lin, B.-B. Wang and P.-F. Yin, Excesses of cosmic ray spectra from a single nearby source, arXiv:1611.09118 [INSPIRE].

[10] T. Abe, J. Kawamura, S. Okawa and Y. Omura, Dark matter physics, flavor physics and LHC constraints in the dark matter model with a bottom partner, JHEP 03 (2017) 058 [arXiv: 1612.01643] [INSPIRE].

[11] S.-J. Lin, X.-J. Bi, J. Feng, P.-F. Yin and Z.-H. Yu, A systematic study on the cosmic ray antiproton flux, arXiv:1612.04001 [INSPIRE]. 
[12] C. Balázs and T. Li, AMS-02 fits dark matter, JHEP 05 (2016) 033 [arXiv:1509.02219] [INSPIRE].

[13] O. Buchmueller, M.J. Dolan and C. McCabe, Beyond effective field theory for dark matter searches at the LHC, JHEP 01 (2014) 025 [arXiv: 1308.6799] [INSPIRE].

[14] C. Arina, E. Del Nobile and P. Panci, Dark matter with pseudoscalar-mediated interactions explains the DAMA signal and the Galactic Center excess, Phys. Rev. Lett. 114 (2015) 011301 [arXiv: 1406.5542] [INSPIRE].

[15] A. Alves, A. Berlin, S. Profumo and F.S. Queiroz, Dark matter complementarity and the $Z^{\prime}$ portal, Phys. Rev. D 92 (2015) 083004 [arXiv: 1501.03490] [InSPIRE].

[16] J. Abdallah et al., Simplified models for dark matter searches at the LHC, Phys. Dark Univ. 9-10 (2015) 8 [arXiv:1506.03116] [INSPIRE].

[17] G. Busoni et al., Recommendations on presenting LHC searches for missing transverse energy signals using simplified s-channel models of dark matter, arXiv:1603.04156 [INSPIRE].

[18] C. Arina et al., A comprehensive approach to dark matter studies: exploration of simplified top-philic models, JHEP 11 (2016) 111 [arXiv:1605.09242] [INSPIRE].

[19] A. Ismail, W.-Y. Keung, K.-H. Tsao and J. Unwin, Axial vector $Z^{\prime}$ and anomaly cancellation, Nucl. Phys. B 918 (2017) 220 [arXiv:1609.02188] [inSPIRE].

[20] J. Kumar and D. Marfatia, Matrix element analyses of dark matter scattering and annihilation, Phys. Rev. D 88 (2013) 014035 [arXiv:1305.1611] [InSPIRE].

[21] V.L. Ginzburg and S.I. Syrovatskii, The origin of cosmic rays, Macmillan, New York U.S.A. (1964).

[22] R. Blandford and D. Eichler, Particle acceleration at astrophysical shocks: a theory of cosmic ray origin, Phys. Rept. 154 (1987) 1 [INSPIRE].

[23] L. Stawarz, V. Petrosian and R.D. Blandford, On the energy spectra of GeV/TeV cosmic ray leptons, Astrophys. J. $\mathbf{7 1 0}$ (2010) 236 [arXiv:0908.1094] [INSPIRE].

[24] F. Aharonian, A. Bykov, E. Parizot, V. Ptuskin and A. Watson, Cosmic rays in galactic and extragalactic magnetic fields, Space Sci. Rev. 166 (2012) 97 [arXiv:1105.0131] [INSPIRE].

[25] A.W. Strong, I.V. Moskalenko and V.S. Ptuskin, Cosmic-ray propagation and interactions in the Galaxy, Ann. Rev. Nucl. Part. Sci. 57 (2007) 285 [astro-ph/0701517] [INSPIRE].

[26] V.L. Ginzburg et al., Astrophysics of cosmic rays, North Holland, Amsterdam Netherlands (1990).

[27] S.-J. Lin, Q. Yuan and X.-J. Bi, Quantitative study of the AMS-02 electron/positron spectra: implications for pulsars and dark matter properties, Phys. Rev. D 91 (2015) 063508 [arXiv: 1409.6248] [inSPIRE].

[28] G. Elor, N.L. Rodd and T.R. Slatyer, Multistep cascade annihilations of dark matter and the Galactic Center excess, Phys. Rev. D 91 (2015) 103531 [arXiv:1503.01773] [InSPIRE].

[29] G. Elor, N.L. Rodd, T.R. Slatyer and W. Xue, Model-independent indirect detection constraints on hidden sector dark matter, JCAP 06 (2016) 024 [arXiv: 1511.08787] [INSPIRE].

[30] J.F. Navarro, C.S. Frenk and S.D.M. White, The structure of cold dark matter halos, Astrophys. J. 462 (1996) 563 [astro-ph/9508025] [INSPIRE].

[31] J.F. Navarro, C.S. Frenk and S.D.M. White, A universal density profile from hierarchical clustering, Astrophys. J. 490 (1997) 493 [astro-ph/9611107] [INSPIRE]. 
[32] M. Cirelli et al., PPPC 4 DM ID: a Poor Particle Physicist Cookbook for Dark Matter Indirect Detection, JCAP 03 (2011) 051 [Erratum ibid. 10 (2012) E01] [arXiv:1012.4515] [INSPIRE].

[33] V.S. Ptuskin, I.V. Moskalenko, F.C. Jones, A.W. Strong and V.N. Zirakashvili, Dissipation of magnetohydrodynamic waves on energetic particles: impact on interstellar turbulence and cosmic ray transport, Astrophys. J. 642 (2006) 902 [astro-ph/0510335] [INSPIRE].

[34] A.W. Strong and I.V. Moskalenko, Propagation of cosmic-ray nucleons in the galaxy, Astrophys. J. 509 (1998) 212 [astro-ph/9807150] [INSPIRE].

[35] I.V. Moskalenko, A.W. Strong, J.F. Ormes and M.S. Potgieter, Secondary anti-protons and propagation of cosmic rays in the galaxy and heliosphere, Astrophys. J. 565 (2002) 280 [astro-ph/0106567] [INSPIRE].

[36] A.W. Strong and I.V. Moskalenko, Models for galactic cosmic ray propagation, Adv. Space Res. 27 (2001) 717 [astro-ph/0101068] [INSPIRE].

[37] I.V. Moskalenko, A.W. Strong, S.G. Mashnik and J.F. Ormes, Challenging cosmic ray propagation with antiprotons. Evidence for a fresh nuclei component?, Astrophys. J. 586 (2003) 1050 [astro-ph/0210480] [INSPIRE].

[38] R. Trotta et al., Constraints on cosmic-ray propagation models from a global Bayesian analysis, Astrophys. J. 729 (2011) 106 [arXiv:1011.0037] [INSPIRE].

[39] K. Auchettl and C. Balázs, Extracting the size of the cosmic electron-positron anomaly, Astrophys. J. $\mathbf{7 4 9}$ (2012) 184 [arXiv:1106.4138] [INSPIRE].

[40] G. Giesen et al., AMS-02 antiprotons, at last! Secondary astrophysical component and immediate implications for Dark Matter, JCAP 09 (2015) 023 [arXiv: 1504.04276] [INSPIRE].

[41] CMS collaboration, Search for dark matter in final states with an energetic jet, or a hadronically decaying $W$ or $Z$ boson using $12.9 \mathrm{fb}^{-1}$ of data at $\sqrt{s}=13 \mathrm{TeV}$, CMS-PAS-EXO-16-037 (2016).

[42] CMS collaboration, Search for narrow resonances in dijet final states at $\sqrt{s}=8 \mathrm{TeV}$ with the novel CMS technique of data scouting, Phys. Rev. Lett. 117 (2016) 031802 [arXiv: 1604.08907] [INSPIRE].

[43] CMS collaboration, Search for dijet resonances in proton-proton collisions at $\sqrt{s}=13 \mathrm{TeV}$ and constraints on dark matter and other models, Phys. Lett. B (2016)

[arXiv: 1611.03568] [INSPIRE].

[44] C. Karwin, S. Murgia, T.M.P. Tait, T.A. Porter and P. Tanedo, Dark matter interpretation of the Fermi-LAT observation toward the galactic center, arXiv:1612.05687 [INSPIRE].

[45] DES, FERMI-LAT collaboration, A. Albert et al., Searching for dark matter annihilation in recently discovered Milky Way satellites with Fermi-LAT, Astrophys. J. 834 (2017) 110 [arXiv: 1611.03184] [INSPIRE].

[46] G. Steigman, B. Dasgupta and J.F. Beacom, Precise relic WIMP abundance and its impact on searches for dark matter annihilation, Phys. Rev. D 86 (2012) 023506 [arXiv:1204.3622] [INSPIRE].

[47] K.G. Begeman, A.H. Broeils and R.H. Sanders, Extended rotation curves of spiral galaxies: Dark haloes and modified dynamics, Mon. Not. Roy. Astron. Soc. 249 (1991) 523 [InSPIRE]. 